\title{
40-Hz coherent oscillations in neuronal systems
}

\author{
Wei Wang, ${ }^{1}$ Guang Chen, ${ }^{1}$ and Z. D. Wang ${ }^{2}$ \\ ${ }^{1}$ Department of Physics and Institute for Solid State Physics, Nanjing University, Nanjing 210093, Peoples Republic of China \\ ${ }^{2}$ Department of Physics, The University of Hong Kong, Pokfulam Road, Hong Kong
}

(Received 5 August 1996; revised manuscript received 11 November 1996)

\begin{abstract}
A characteristic coherent oscillation with a frequency of $40 \mathrm{~Hz}$ which relates to some functions of the brain is shown to be intrinsic due to the nonlinear firing of spikes of neurons when the network is situated in a stimulus-induced oscillatory state. This oscillation mode is associated with a synchronized firing pattern, and is robust to a variety of couplings between the neurons and to a wide range of external stimuli. [S1063-651X(97)05409-3]
\end{abstract}

PACS number(s): 87.10. $+\mathrm{e}$

Recently, considerable attention has been drawn to the existence of coherent synchronized oscillatory activity in many neuronal systems. It has been found experimentally that large-scale synchronized neuronal activity is often accompanied by oscillatory firing patterns with a frequency about $40 \mathrm{~Hz}$ (in the $\gamma$ band, usually referred to as $40-\mathrm{Hz}$ oscillations) [1]. For example, in the cat's visual cortex and in the awake (or in rapid-eye-movement sleep) state of humans, coherent synchronized oscillatory responses have been observed through local field potential and multiunit activities [1]. It has also been shown that the synchronization probability depends on the configuration of visual stimuli and that it does reflect some of the gestalt criteria that are used for scene segmentation [1]. Therefore this kind of long-distance synchronized spatiotemporal dynamical behavior has been suggested as a mechanism for the binding of spatially distributed features into a coherent object [1-3]. The neurons which process different features of the same object oscillate with a fixed phase, while neurons which code different objects oscillate with different phases or at random.

To date, there have been some theoretical studies on the above mentioned coherent oscillations; these are based either on a model of coupled oscillators or a model of integrate and fire; few of them have taken into account the detailed features of the neuronal activity, such as its recovery or its adaptation after the firing of spikes. Although a completely synchronized oscillation with zero phase difference may be obtained in these models, all the couplings are assumed to be excitatory [4], an assumption which seems to be oversimplified and which may not be consistent with the real situation. In particular, the biophysical origin of these coherent oscillations and their relationship with the synaptic interaction (or coupling), as well as with the external stimuli, are not well studied. In addition, the frequency spectrum of the oscillations has rarely been addressed theoretically, and the question of how the synchrony is established and what role the oscillatory firing plays in the dynamical informational processes of a neuronal system is not well understood.

In this paper, we attempt to address the above issues. We show that a biologically relevant coupled neuronal network naturally displays a coherent synchronized oscillation with a characteristic frequency of about $40 \mathrm{~Hz}$ for a wide range of stimuli, as well as for various synaptic couplings. The system exhibits a synchronized spatiotemporal pattern of firing which is established by reciprocal connections including excitatory and inhibitory couplings among spatially distributed neurons. It also appears that these oscillations are an intrinsic dynamical phenomenon in a neuronal system as long as the interaction between the neurons is of long range.

Experimentally, neurons always show irregular or noiselike activity in various situations, even in spontaneous firing states. In our earlier work [5], we studied the firing of a single Hindmarsh and Rose (HR) neuron [6] in a modified Fitzhugh neuronal model [7]. It was found that as the stimulus $I$ increases, the system undergoes a period-adding firing state (for $1.3<I<2.9$ ), a chaotic firing state and a reversed period-doubling cascade (for $2.9<I<3.5$ ), and a highfrequency repetitive firing state (for $I>3.5$ ) [5]. Except for the high-frequency repetitive firing, which is a simple linear firing and may have little neurobiological relevance, the neuron shows a complex firing pattern of bursts of spikes, the number of spikes and the time interval between spikes in the burst being fixed (for $1.3<I<2.9$ ) and fluctuating (for $2.9<I<3.4)$ due to the nonlinearity in the system. The duration or the interval of bursts ranges from 32 to $14 \mathrm{~ms}$ for $1.3<I<3.5$ (cf. Fig. 1 in Ref. [5]) using a time scale defined later in the text. This corresponds to a frequency in a range of 32 to $70 \mathrm{~Hz}$. How can a network consisting of such neurons exhibit coherent synchronized oscillations with a characteristic frequency of $40 \mathrm{~Hz}$ ?

To answer this very interesting and nontrivial question, we start with the HR neuronal model [6] and construct a network by the following equations $[5,8]$ :

$$
\begin{gathered}
\frac{d X_{i}}{d t}=Y_{i}-a X_{i}^{3}+b X_{i}^{2}-Z_{i}+I_{i}+\frac{1}{N} \sum_{j=1, j \neq i}^{N} J_{i j} S_{j}(t), \\
\frac{d Y_{i}}{d t}=c-d X_{i}^{2}-Y_{i}, \\
\frac{d Z_{i}}{d t}=r\left[s\left(X_{i}-X_{0}\right)-Z_{i}\right],
\end{gathered}
$$

where $a=1.0, b=3.0, c=1.0, d=5.0, s=4.0, r=0.006$, and $X_{0}=-1.6$. Each neuron is characterized by the membrane potential $X_{i}$, the recovery variable $Y_{i}$, and a slow adaptation current $Z_{i}$. The external stimuli are given by $I_{i}$ 


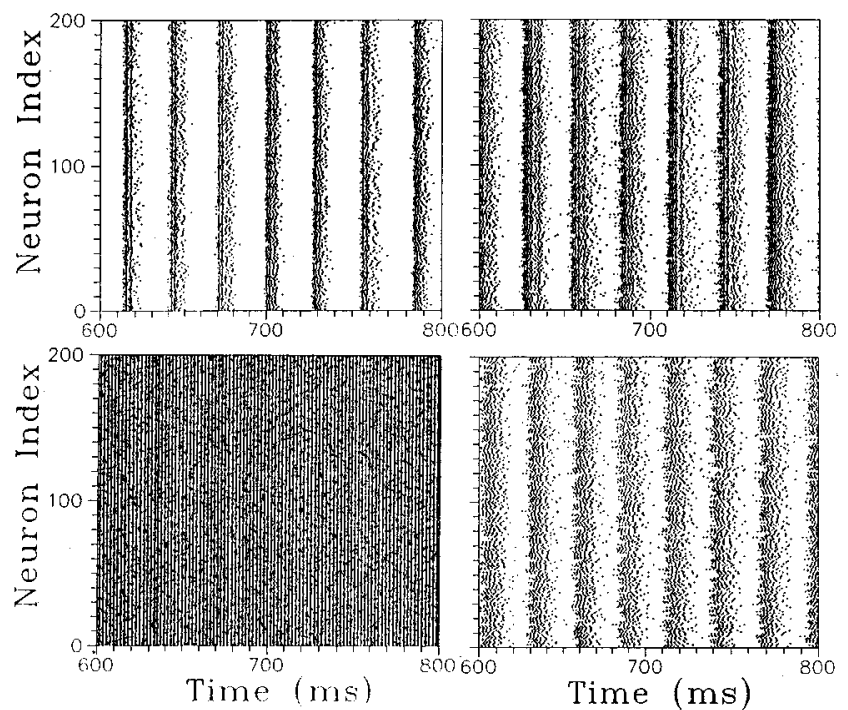

FIG. 1. Spatiotemporal pattern of the firing of spikes for a network with a constant stimuli $I_{i}$ and uniformly distributed couplings $J_{i j}$. (a) $I_{i}=2, J_{i j} \in[-5,10]$ (upper left); (b) $I_{i}=3, J_{i j} \in[-5,10]$ (upper right); (c) $I_{i}=5, J_{i j} \in[-5,10]$ (lower left); (d) $I_{i}=3, J_{i j}$ $\in[-3,5]$ (lower right)

and are limited to be $I_{i}>1.3$. The effect of the firing activity of the $j$ th neuron on the $i$ th neuron is modeled by a synaptic current $J_{i j} S_{j}(t)$, generated when the $j$ th neuron is active, i.e., $S_{j}(t)=\theta\left(X_{j}(t)-X^{*}\right)$ where $X^{*}$ is a membrane potential threshold and $\theta(x)=1$ if $x \geqslant 0$ and $\theta(x)=0$ if $x<0$. If the global interaction strength $J_{i j}>0$ (an excitatory coupling between neurons), there is a positive excitatory postsynaptic potential (EPSP). In a real neuronal system there is always a kind of inhibitory coupling with $J_{i j}<0$ which produces a negative inhibitory postsynaptic potential (IPSP), and it is the spatial and temporal summation of these synaptic potentials, both EPSP and IPSP, on a given neuron that results in the firing or nonfiring of spikes. Therefore, to be more realistic, we include also an inhibitory coupling in the network. The coupling strengths $J_{i j}$ are assumed to be randomly distributed in a certain range, and to take both positive and negative values. We also assume that the network is situated in a stimulus-induced oscillation state, i.e., $I_{i}=$ const. Our simulations are performed by using a modified fourth-order Runge-Kutta method. The time scale is chosen in such a way that the width of a spike numerically obtained from the above model equations is consistent with its counterpart neurobiological experimental result, which is about $2 \mathrm{~ms}$.

Figure 1 shows the spatiotemporal patterns of neuronal activities. The network consists of 200 neurons stimulated by the same input $I_{i}$. The coupling strengths are uniformly distributed, $J_{i j} \in[-5,10]$, which means that the percentage $\eta$ of the inhibitory coupling is $33 \%$ (or $\eta=33 \%$ ) of the total number of the synaptic interactions. We can see that when $I_{i}<4$, the whole system exhibits a cooperative dynamical phenomenon; the neurons fire synchronously and the spatiotemporal behavior shows oscillatory firing patterns [see Figs. 1(a) and 1(b)]. The two patterns are similar, and the synchronization extends to the entire network. Bursts of spikes with a duration of 10-12 ms occur for all the neurons, followed by a quiescent interval, about $12-20 \mathrm{~ms}$, after the

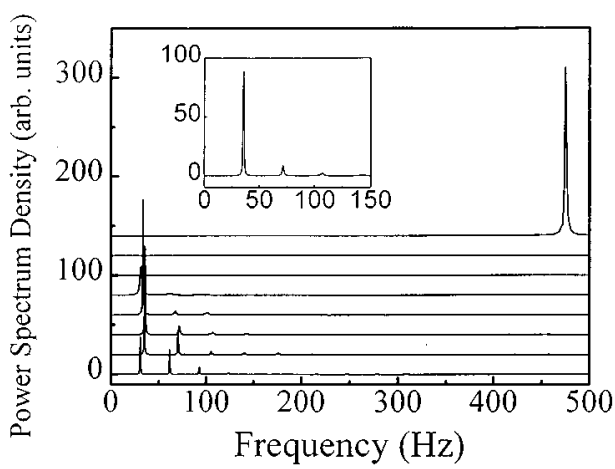

FIG. 2. The power spectrum density of the average synaptic current $\widetilde{I}_{\text {syn }}$. The value for each curve has been shifted by a factor of 20 on the vertical axis. From the bottom to the top, the values of $I_{i}$ are $1.5,2.0,2.5,3.0,3.5,4.0,4.5,5.0$, respectively. The inset shows the case for $I_{i}=2.5$.

burst. The total period is about $24-31 \mathrm{~ms}$, which gives a frequency of 32-42 Hz. By contrast, when $I_{i}>4$, the system exhibits a repetitive firing state; the pattern shows a nearly periodic firing with a high frequency; e.g., in Fig. 1(c) with a frequency $f \approx 500 \mathrm{~Hz}$. For comparison, a pattern for a network with $J_{i j} \in[-3,5]$ is plotted in Fig. 1(d), where we also see the same synchronized $40-\mathrm{Hz}$ oscillations.

In Fig. 2 we show the power spectrum density of the mean synaptic current, $\widetilde{I}_{\text {syn }}(t)=N^{-2} \sum_{i, j} J_{i j} S_{j}(t)$ for different stimuli $I_{i}$. This average of synaptic activity is associated with the local field potential. We can see clearly that when $I_{i}<4$ there is a sharp peak around $40 \mathrm{~Hz}$, whereas for $I_{i}>4$, the peak shifts to a high frequency even as high as 500 Hz. Physically, the above mentioned behavior can be understood as follows. The uncoupled neurons fire with a frequency in the range $32-70 \mathrm{~Hz}$ if the stimulus is in the nonlinear firing region $1.3<I_{i}<3.5$. On the other hand, they fire with a frequency $f>166 \mathrm{~Hz}$ when the stimulus is in the simple linear firing region $I_{i}>3.5$, where the neurons show a repetitive periodic firing of spikes with a period $T<6 \mathrm{~ms}$ (cf. Fig. 1 in Ref. [5]). In the presence of coupling, the stimulus for a neuron is effectively changed from $I_{i}$ to $I_{i}^{\prime}=I_{i}+\widetilde{I}_{\text {syn }}(t)$. Simulations show that $\widetilde{I}_{\text {syn }}(t)$ is periodic, with an amplitude that oscillates between 0 and 1.2 when $I_{i}<4$ and 0 and 2 when $I_{i}>4$. The time average $\left\langle\widetilde{I}_{\text {syn }}(t)\right\rangle$ is found to be $0.13,0.22$, and 0.46 , for $I_{i}=2,3$, and 5 , corresponding to Figs. 1(a)-1(c), respectively. Thus the effective stimuli are $I_{i}^{\prime}=2.13,3.22$, and 5.46 in these cases. For the former two values the system exhibits the $40-\mathrm{Hz}$ oscillations because the effective stimulus satisfies $I_{i}^{\prime}<3.4$. For the last case, the system will exhibit a high-frequency repetitive oscillation since $I_{i}^{\prime}>3.5$.

As we have seen in the simulations, if the neurons (or most of them) are in the nonlinear firing state, the $40-\mathrm{Hz}$ component is not sensitive to the specific choice of the stimuli and the coupling strengths. We have checked this point by choosing different values of the stimuli and different distributions of the coupling strength. In Fig. 3, we plot the frequency of the main peak versus the stimulus $I_{i}$ for three different distributions of the coupling strength. The inset shows the $40-\mathrm{Hz}$ oscillation in detail. One can see clearly that for all the cases, the $40-\mathrm{Hz}$ oscillation occurs in a range 


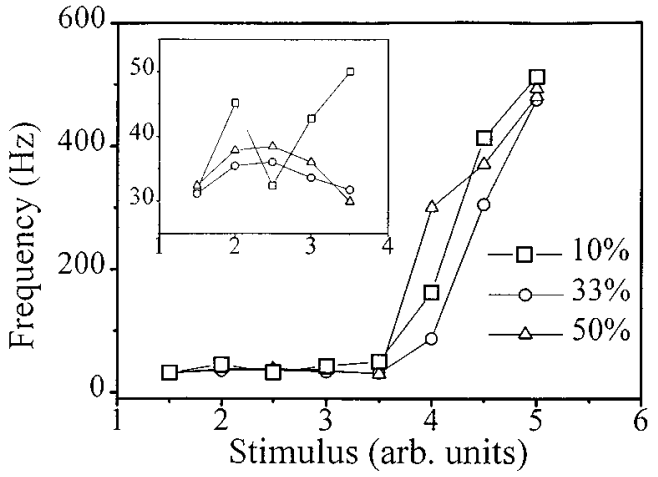

FIG. 3. The oscillation frequency versus the stimulus for three different uniform distributions of couplings $J_{i j}$ with the percentage of the inhibitory coupling $\eta$ : (a) $J_{i j} \in[-1,10], \eta=10 \%$; (b) $J_{i j}$ $\in[-5,10], \eta=33 \%$; (c) $J_{i j} \in[-10,10] \eta=50 \%$. Inset: the $40-\mathrm{Hz}$ range in detail.

of stimulus $\left(I_{i}<4\right)$, but disappears outside this range. We have also performed simulations for cases of nonuniformly distributed coupling strengths. The results obtained show the same characteristics. Notice that the $40-\mathrm{Hz}$ oscillatory state survives unless we impose significant inhibitory coupling, typically $\eta>60 \%$ of the inhibitory coupling is required to exclude it. For example, with $J_{i j} \in[-10,10]$ and $\eta=50 \%$, we see that the firings consist of some small clusters and the bursts do not all occur at the same time; but there is still a rough period of $25 \mathrm{~ms}$ for a range of stimuli, which leads to the $40-\mathrm{Hz}$ oscillations. Also note that, when $\eta$ increases, the height of the main peak in the spectrum decreases, the range of the $40-\mathrm{Hz}$ oscillations becomes narrow, and the oscillation frequency shifts to a higher value. In addition, when $\eta=0$ (or $J_{i j}>0$ ), e.g., $J_{i j} \in[0,5]$, we find that the peak of $40-\mathrm{Hz}$ oscillations in the spectrum is much lower than that of the cases of $\eta \neq 0$ due to all the synaptic input being excitatory.

It is worth pointing out that, even if we include the effects of the short-time delay between the couplings, a weak background noise, or some distribution of the stimuli, the neuronal activity in the network still oscillates with a frequency around $40 \mathrm{~Hz}$ [9]. We may conclude therefore that the synchronized $40-\mathrm{Hz}$ oscillations result from the the nonlinear firing of spikes of the neurons. These oscillations are an intrinsic dynamical phenomenon of the network. However, the dynamics of the oscillations is complex. When $I_{i}<2.6$ (or $\left.I_{i}^{\prime}<2.9\right)$ the number of spikes and the interval between spikes in the bursts are fixed; the neurons in the network show a periodic behavior [see Fig. 1(a)]. Whereas for $2.6<I_{i}<3.5$ (or $2.9<I_{i}^{\prime}<4$ ) the neurons in the network show chaotic firing, the number of spikes and the interval between the spikes fluctuate, and also the onset times of bursts fluctuate as $\eta$ increases [see Figs. 1(b) and 1(d)]. We plan to discuss this synchronized chaotic nature elsewhere [9].

Now, let us turn to the establishment of the synchrony of the firing of spikes in the network. Obviously, if all neurons are stimulated by an identical value of $I_{i}$ and the values $J_{i j}$ are set to be the same for all connections, the network will be synchronized completely, with zero phase difference between the firing of neurons. In this case, the neurons respond to a pulse interaction simultaneously due to the symmetry

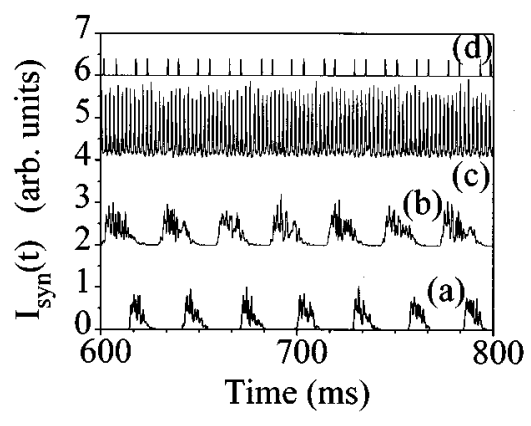

FIG. 4. The curves (a)-(c) represent the averaged synaptic currents $\widetilde{I}_{\text {syn }}(t)$ as functions of time, corresponding to Figs. 1(a)-1(c) [for curves (b) and (c), the values of 2 and 4 are shifted on the vertical axis, respectively]. Curve (d) shows the case of the synchronization with zero phase difference for $I_{i}=3$ and $J_{i j}=0.4$ (shifted by a value of 6 on the vertical axis).

and the uniformity of the stimulus and the coupling. In other words, the neurons reach their threshold values at the same time, and their effective stimuli have the same values. These enable the neurons to fire synchronously with zero phase difference. Introducing a distributed coupling $J_{i j}$ implies a destruction of this symmetry of interaction and brings some disorder into the system. Generally, the dynamical behavior of an individual neuron is governed mainly by the stimulus exerted. However, the firing of spikes is modulated by the interaction, and the whole system relaxes into a coherent state. Whether this state is synchronized or not depends on how strong the introduced disorder is, and on what extent the symmetry is broken. Presumably, this synchronized state is not of zero phase difference between the firing of neurons. After a burst of spikes, a neuron returns to its rest potential, and then experiences a summation of the synaptic input from other neurons extended over a considerable spatial and temporal extent. Once the effective stimulus reaches the threshold value, the neuron will produce another burst of spikes. Since different neurons may have different effective stimuli and thus require different times to reach the threshold values, the neurons will show different behaviors, i.e., there may be a little delay between the firings of bursts among the neurons. As a result, the firings between neurons show a synchronization but with some phase differences. On the other hand, as we knew previously, the averaged effective stimulus $I_{i}^{\prime}=I_{i}+\widetilde{I}_{\text {syn }}(t)$ is periodic and not pulselike, due to the nonlinear spatial and temporal summation of the synaptic input. It is this periodic stimulus, a mean field action, that modulates the system to fire the bursts roughly in synchronization on a large scale. Consequently, bursts of spikes will show an oscillatory firing pattern which is synchronized over the whole system. As a matter of fact, when the nonuniformity introduced is significant, the system will lose the feature of synchronized oscillatory firing. In order to see the mechanism described above more clearly, we plot the spatial average of the synaptic current against the time in Fig. 4. Clearly, the periodic feature relates to the oscillation behavior of the spatiotemporal pattern shown in Fig. 1. The case of synchronization with zero phase difference is also shown.

Finally, we remark on the role the $40-\mathrm{Hz}$ oscillations play in the functions of the brain. Psychophysical research results indicate that scene segmentation and the binding of the fea- 
tures of complex objects can be accomplished within 100$200 \mathrm{~ms}$ [10]. If this function is realized by the synchronized oscillatory activity, a sufficient number of oscillatory cycles must be accommodated in this time span. We find that the $40-\mathrm{Hz}$ synchronized oscillations could be formed within short time, and then experience 3-6 cycles within 100-200 $\mathrm{ms}$ [9]. Oscillatory activity, however, with frequencies in the $\alpha(8-13 \mathrm{~Hz})$ to $\beta(14-30 \mathrm{~Hz})$ range, or faster than $80 \mathrm{~Hz}$, is not suitable for facilitating long-range synchrony $[9,11]$. Accordingly, oscillations with $\gamma$ frequencies seem to be most appropriate for the establishment of sensory representations $[11,12]$.

In conclusion, coherent synchronized intrinsic oscillations with a characteristic frequency of $40 \mathrm{~Hz}$ in a global coupled neuronal network have been elucidated. These oscillations are due to the internal dynamics of the neurons modulated by the nonlinear spatial and temporal summation of firing of spikes, and are robust to a wide range of the external stimuli and to various forms of the distribution of synaptic interaction parameters.

We are grateful to Professor P. K. Mackeown for his critical reading of the manuscript. One of the authors (W. Wang) acknowledges support in part by the National Laboratory of Solid State Microstructure, the Young Research Foundation of the Committee of National Education and the Outstanding Young Research Foundation of the National Natural Science Foundation.
[1] C. M. Gray, P. Konig, A. K. Engel, and W. Singer, Nature (London) 338, 334 (1989); R. Eckhorn et al., Biol. Cybern. 60, 121 (1988); C. Skarda and W. J. Freeman, Behav. Brain Sci.. 10, 161 (1987); R. R. Llinas, A. A. Grace, and Y. Yarom, Proc. Natl. Acad. Sci. USA 88, 897 (1991); M. A. Whittington et al., Nature (London) 373, 612 (1995); A. K. Engel, P. Konig, and W. Singer, Proc. Natl. Acad. Sci. USA 88, 9136 (1991); 92, 290 (1995); Ch. von der Malburg and W. Schneider, Biol. Cybern. 54, 29 (1986); O. Sporns, G. Tononi, and G. M. Edelman, Proc. Natl. Acad. Sci. USA 88, 129 (1991).

[2] F. Crick and C. Koch, Seminars Neurosci. 2, 263 (1990).

[3] C. M. Gray and W. Singer, Proc. Natl. Acad. Sci. USA 86, 1698 (1989).

[4] R. E. Mirollo and S. H. Strogatz, SIAM (Soc. Ind. Appl. Math.) J. Appl. Math. 50, 1645 (1990); M. Tsodyks, I. Mitkov, and H. Sompolinsky, Phys. Rev. Lett. 71, 1280 (1993); U.
Ernst, K. Pawelzik, and T. Geisel, ibid. 74, 1570 (1995).

[5] Wei Wang, G. Perez, and H. A. Cerdeira, Phys. Rev. E 47, 2893 (1993).

[6] J. L. Hindmarsh and R. M. Rose, Nature (London) 296, 162 (1982); Proc. R. Soc. London, Ser. B 221, 87 (1984); 225, 161 (1985).

[7] Wei Wang, J. Phys. A 22, L627 (1989); Commun. Theor. Phys. 18, 265 (1992).

[8] D. Hansel and H. Sompolinsky, Phys. Rev. Lett. 68, 718 (1992).

[9] Wei Wang et al. (unpublished).

[10] I. Biederman, in Visual Cognition and Action, edited by D. N. Osherson, S. M. Kosslyn, and J. M. Hollerbach (MIT Press, Cambridge, MA, 1990), pp. 41-72.

[11] W. Singer and C. M. Gray, Annu. Rev. Neurosci. 18, 555 (1995).

[12] J. J. Hopfield, Nature (London) 376, 33 (1995). 